\title{
THE MULTI-AGENT APPROACH FOR DEVELOPING A CYBER-PHYSICAL SYSTEM FOR MANAGING PRECISE FARMS WITH DIGITAL TWINS OF PLANTS
}

\author{
Vladimir Laryukhin, Petr Skobelev \\ Samara State Technical University \\ Russia \\ vladimir.larukhin@live.ru, petr.skobelev@gmail.com
}

\author{
Oleg Lakhin, Sergey Grachev \\ Knowledge Genesis Group \\ Russia \\ lakhin@kg.ru,grachev@kg.ru
}

\author{
Vladimir Yalovenko, Olga Yalovenko, \\ Peschanokopskaya Agrarian Laboratory \\ Russia \\ vladimiryalovenko@mail.ru,olyayalovenko@gmail.com
}

Article history:

Received 10.12.2019, Accepted 26.12.2019

\begin{abstract}
The paper presents the multi-agent approach for developing cyber-physical system for managing precise farms with digital twins of plants. It discusses complexity of the problem caused by a priori incompleteness of knowledge about factors of plant growth and development, high uncertainty of crops cultivation, variety of weather, business and technical requirements, etc. The approach proposes knowledge bases and multi-agent technology in combination with machine learning methods for designing considered systems. Digital twin of plant is specified as an agent based on ontology model of objects relevant for plant cultivation (specific sort of plant, soil, etc) associated with history of operations and environment conditions. The architecture and functions of system components are designed. The expected results of system implementation and the benefits for farmers are discussed.
\end{abstract}

\section{Key words}

Cyber-physical system, multi-agent system, knowledge base, digital twin, plant cultivation, precise farming.

\section{Introduction}

Cyber-physical systems (CPS) is a new type of systems integrating computation, communication and control components, including sensors, actuators and network connectors [Rajkumar et al., 2010]. Starting from embedded systems, CPS now are evolving as "smart systems" with fusion and interaction of real and virtual worlds, which can provide growing level of autonomous behavior with recognition of patterns, decision making and collaboration with users.

Multi-agent systems (MAS) are considered as a powerful tool for bringing a number of smart-* features to CPS [Leitao et al., 2016]: "MAS technologies share common ground with CPS and can empower them with multitude capabilities in their efforts to achieve complexity management, decentralization, intelligence, modularity, flexibility, robustness, adaptation, and responsiveness".

The concept of digital twin was initially associated with product life-cycle in manufacturing [Tao et al., 2018]. For example, for aerospace vehicle "digital twin is a kind of ultra-high fidelity simulation integrating with an on-board health management system, maintenance history, and historical vehicle and fleet data. It can mirror the whole life of a specific flying physical twin (or tail number), which enables significant gains in safety and reliability". Big data analytics and digital twins are considered as a basis for smart manufacturing [Qi and Tao, 2018].

Research subject of this paper is the key requirements, functionality and architecture of cyber-physical multiagent system (CP MAS) for precise farm management. As a part of the designed system digital twins of plant will be introduced. Digital twin will be designed as computer model of plant growth and development based on available domain knowledge and various data acquired during the process of plant cultivation. Ontologydriven knowledge base, multi-agent technology and machine learning methods will be proposed for creating and maintaining digital twin of plants for mirroring state and 
parameters of real plants and using the difference in a cycle of farms resource management for application in wheat production.

\section{Problem Statement}

Modern precision farming technologies with daily controlled plant cultivation can significantly improve the quality of products and efficiency of crop production [Dutta et al., 2017].

However, today precise farming meets a number of challenges with complexity of the technology and a priori incompleteness of knowledge about factors of plant growth and development, high uncertainty of crops cultivation, variety of weather, business and technical requirements, etc. In contrast with machine assembly, in crop cultivation there is no direct, well-developed technological process, it is difficult to plan operations in advance and high adaptability is required. Each crop, each farm and each season is unique and requires specific knowledge about biological, chemical and physical processes in plants and soils. As a result, in one season a farmer can have good harvest without big costs, whereas in another, for example, with high expenses on fertilizers, he can't get any harvest at all under the same weather conditions. The problem becomes even more complex with the growing climate changes.

Under these circumstances, agronomists have to constantly monitor many fields with different plants and control the difference between ideal and real crop development. In case of a gap, they have to come up with ideas on how to make changes in the agro-technology, adaptively modifying schedules for precise machines depending on behavior and reaction of plants to different factors and changes of weather.

Despite the big progress with new varieties of plants and agro-technologies, satellites and drones, electronic maps and hyper-spectrum image processing, precise machines and other technival advances, this decision-making process is still not automated and is very agronomist-centric, knowledge- and experiencedependable, informal and manual, time-consuming and expensive, and very risky for business and farm owners.

\section{Proposed Approach to Solving of Problem}

Solution of the problem could be achieved by developing CP MAS for farm management integrating sensors, actuators and communication tools, and, on the other hand, computer models of plant growth and development combined with knowledge base and decisionmaking tools for farmers.

The conceptual model of CP MAS is proposed in Fig. 1. The key feature of CP MAS for plant cultivation should be high adaptability, which makes it possible to quickly, flexibly and efficiently review and adjust plans of plant development, providing everyday sup- port and recommendations to agronomists and farmers in decision-making.

Let us consider the key technologies which will be developed for CP MAS.

\subsection{Collective Decision Making Based on Multi- Agent Technology}

The main idea of the proposed approach is to consider crop cultivation as a complex adaptive system with distributed collective decision making among crop varieties, soil and fertilizers, precise machines, etc. [Rzevski and Skobelev., 2014].

The hypothesis of this approach is that the reasoning of agronomist and other specialists in farm management could be modelled as a process of above entities selforganization which can be implemented with the use of multi-agent technology.

Agents are defined as autonomous software objects able to react to events, make their own decisions and interact with each other for coordination of decisions. In the developed approach agents form demand-resource networks (DRN) and operate on internal virtual market with the opportunity to buy and sell time slots in the schedule. During this process agents are able to discover problems and solve conflicts applying protocols of agent negotiations and compensation method based on individual functions of satisfaction and bonus-penalties of agents.

In case of precise farm management, several new classes of DRN agents can be introduced:

1. Agent of farm - to maximize objectives given by farm owner;

2. Agent of plant - to maximize plant productivity and efficiency;

3. Agent of soil - to maximize richness of microelements and water in soil;

4. Agent of fertilizer - to maximize quality of crops, etc.

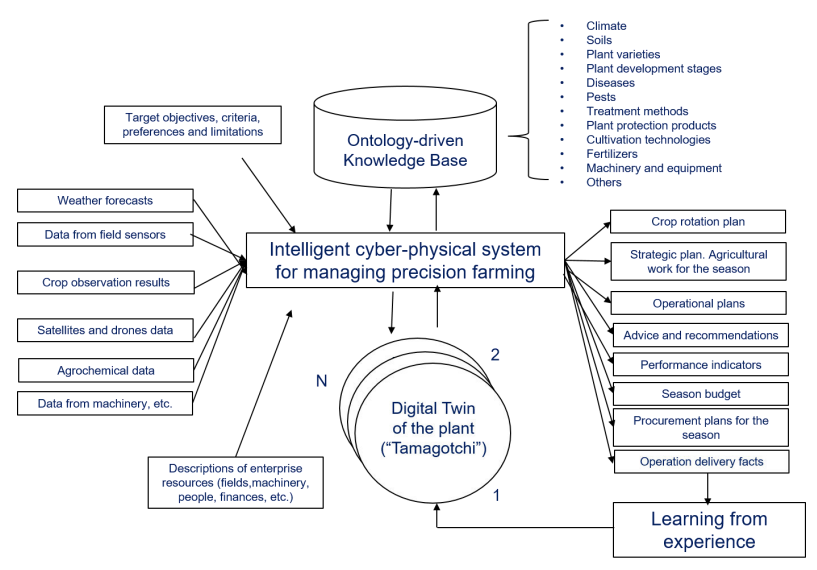

Figure 1. CP MAS for precision agriculture with digital twins of plants: the input-output data. 
As a results the plan for crop cultivation will be formed in virtual market of CP MAS and then adaptively changed by events in a bottom-up way.

The state of the schedule can be considered as "unstable equilibrium" reflecting balance of agents interests for each concrete situation.

The function of satisfaction for agent of plant " $i$ " could be the following:

$$
y_{i}^{P l n t}=\sum_{k P}^{K P} f_{k P}^{P \operatorname{lnt} i}\left(x_{k P}^{P \ln t i}, x_{k P}^{P l n t i d}\right)
$$

where $x^{i d}$ is ideal value for plant, $x^{i}$ is current value for plant, $f_{k P}^{P l n t i}$ is $k P$-th component of plant function (productivity, costs, etc), $K P$ is the number of components.

In case of event, each option can be computed as a delta of satisfaction function:

$$
\Delta=y_{i}^{P l n t}(S 1)-y_{i}^{P l n t}(S 2)
$$

where $S 1$ is the current state and $S 2$ is the new state in case of new decision.

The most efficient will be the decision:

$$
\frac{\Delta}{\text { Cost }} \rightarrow \max
$$

where Cost is the sum of Physical Costs and Decision Making Costs on virtual market.

The satisfaction function for agents of soil, fertilizers and others can be specified in the same way.

Functions of penalties and bonuses are given to compute how much it will cost to change value of function of satisfaction.

As a result the $y^{\text {Farm }}$ will grow by solving conflicts among involved agents but also because of investments by farm agent.

Farm agent can support agents which will worsen their state in order to improve the total satisfaction of farm $y^{\text {Farm }}$.

The discussed software agents can be further specified in more details by their own objectives, models of behavior and available resources, as well as by methods for discovering and resolving conflicts. It will bring the so called "Emergent Intelligence" in CP MAS able to demonstrate nondeterministic behavior of the system with various non-linear phenomena of complex adaptive systems, relevant for plant cultivation.

Agents, represented by computer programs, working in interests of plants and other entities can be used as digital twins with the ability to use domain knowledge and learn through experience.

\subsection{Ontology and Base of Knowledge for Decision Making Support}

Ontology is defined as a formal specification of domain knowledge which can be used for agents decision making [Rzevski and Skobelev., 2014].
Today this knowledge and experience is accumulated exclusively by agronomists during the whole life. Usually such knowledge is hidden and not formalized, fragmented and difficult for verbalization, transfer and utilization in decision making.

The ontology of precision farming could be specified by the following basic topics for main classes of concepts and relations: climate, soils, plant varieties, plant development stages, diseases, pesticides, treatment methods, insects, plant protection products, cultivation technologies, fertilizers, machinery and equipment and others. Instances of these classes form the enterprise knowledge base.

Semantic networks of these concepts and relations and their instances can be applied by agents for finding relevant ways of problem solving. For example, in case of specific insect, the plant agent can find and propose required pesticides and machines.

In case of wheat, for example, more than 100 phases of plant development have been studied. And it is already partially known at what stage wheat makes its decisions. Thus, if at one stage wheat decides that the summer will be dry, it determines to grow a low stem, then no further steps, such as fertilizing, will be able to change this wheat decision.

With the use of ontologies separated from source code of agents one can add new knowledge for decision making without full reprogramming of CP MAS.

\subsection{Data Mining for Agents of Digital Twins of Plants: Learning from Experience}

The big data of each farm, including actions, effects and responses, as well as many other parameters describing the context of the current season and the specific situation of each day (temperature, accumulated precipitation, etc.), must be stored on a daily basis and constantly analyzed in order to produce a continuously updated forecast, reducing the degree of uncertainty and risks for farmers.

Data mining methods and tools [Rzevski and Skobelev., 2014] can be applied to discover new knowledge in this data and automatically correct and refine computer programs of agents, comparing results of planned and actual operations.

The examples of data which can be regularly collected in modern farms include the following:

1. samples are regularly taken for agrochemical soil analyzes;

2. long-term and short-term forecasts of weather changes are built daily, including precipitation, soil temperature, etc.;

3. every day notes are received from agronomists in fields: phases of plant development, increase in leaf size, dangerous insects, threat of plant diseases, etc.

4. detailed history of each field processing, including plan and fact information on results of agricultural operations with the use of specific machines, fertilizers and pesticides; 
5. satellite and drone images of fields with the use of hyper-spectrum cameras;

6. GPS tracking of machines and parameters of agro operations, etc.

This data associated with stages of plants growth and developments will be generalized for adjustment of digital twins of plants, for example, with the use of methods and tools of clustering or any other technologies. It will help to specify new rules for ontology and knowledge base of farm which agents can apply in their automatic decision making.

Resulting clusters or patterns can be combined with ontology-driven reasoning for decision making support.

\subsection{Digital Twins of Plants}

The combination of above mentioned technologies can provide opportunity for developing software agents working as digital twins of real plants:

1. at any moment of time agent of plant represents and reflects current stage of plant growth and development;

2. agent of plant can be used for "what-if" games to form the best possible options in plant cultivation;

3. agents of plants learn from everyday experience.

Digital twin of plant will be created for each field to mirror real plant growth and development (as a "tamagotchi" toy). It would mirror plant development on everyday basis, representing the most predicted version of the plant development plan, updated daily with data from the weather server, sensors in fields, observations of agronomists, etc.

Thus, before the agronomist makes suggestions on the actions that need to be taken on each field in a given situation, he can "simulate" the impact on the crop and analyze its possible "response".

This process needs to be supported in the proposed system so that the agronomist can make offers to plant, and it will respond with its reaction, which can be then compared with the response of the real plant. In this way knowledge on plant cultivation can be adjusted year after year, modifying the decision-making model of plant and creating a more accurate "digital twin" expanding a possible state graph of its agent under various conditions.

\section{Functionality and Architecture of the Proposed System}

Using ontologies and agents in combination of data mining one can make planning of everyday operations in CP MAS but also play "What if" games and form the most perspective scenarios for the season, forecasting yields and risks for business.

For example, with the help of CP MAS agronomists can make such decisions:

1. what crops to plant in the new season and in which fields, as well as which fields should be left under steam;

2. when to start sowing or harvesting;

3. whether plant nutrition or protection is required;

4. under what weather conditions tractors to go around fields and what agrochemical requirements must be maintained (speed, etc.);

5. what equipment should apply plant protection products, with what process parameters, etc.

The basic architecture and functionality of an CP MAS with "digital twins" of plants are shown in Fig. 2 and Fig. 3.

The main components include digital IoT platform which provide data and commands to farm workers, CPMAS for decision making support, digital twin of plant, simulation and forecasting modules.

The digital twin of a plant can be used both in making operational decisions on each field but also for "what if" modeling of situations, as well as forecasting and risk assessment.

The main components include pattern recognition of situations and ontology editor and editor of plant model, which provide farm planning. But also this model can be loaded to simulator for what-if games or to module for adjusting existing model if it becomes inadequate.

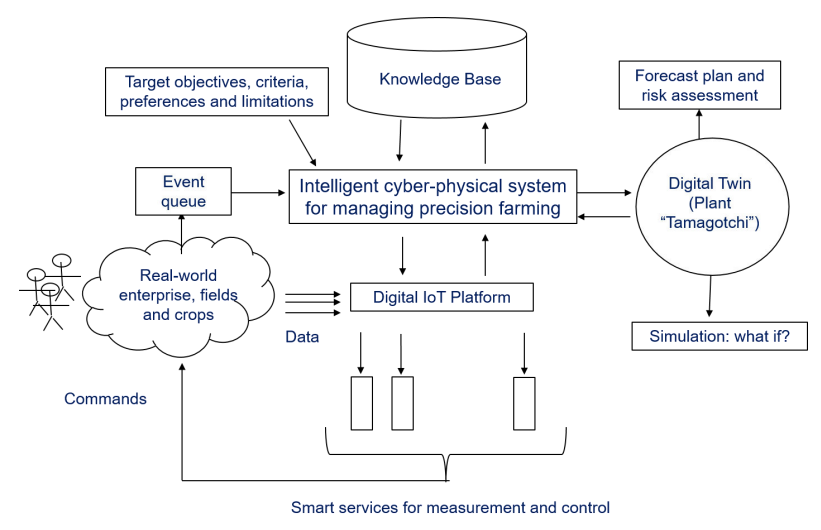

Figure 2. The architecture of CP MAS with digital twins of plants

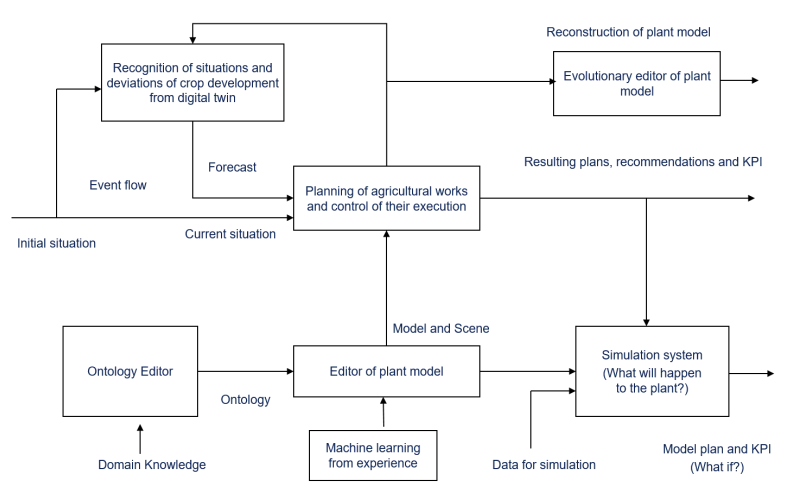

Figure 3. Functionality of CP MAS with digital twins of plants 
The functionality of CP MAS will cover the following features:

1. Support the knowledge base with domain ontology.

2. Gather and formalize available domain knowledge for plant cultivation determining behavior of plant, for example, for wheat cultivation.

3. Support design of agents representing "digital twins" of plants for making decisions.

4. Collect examples of life situations where plants behave in the most predictable and, alternatively, completely unpredictable way.

5. Recognize patterns of problem situations and generate recommendations for solving problems.

6. Provide methods and tools of collective decisionmaking for planning and scheduling of farm resources.

7. Provide data mining methods and tools for learning from experience.

8. Enable the agronomist to make simulations and adjust parameters of the digital twin of plant to compare with real plant growth.

9. Provide access to CP MAS for farmers and agronomists via mobile phone.

When working with the digital twin of plant, it must be possible to make direct requests for predicting plant development in the near future but also give "reverse" ones: what needs to be done to still have chance to increase crop yield - for example, what extra nutrition is needed and what types of fertilizers can be used?

\section{Conclusion}

This paper describes the initial requirements, the functionality and architecture of the CP MAS with digital twins of plants. The key idea of the developed approach is to support decision making of farmers by specifying domain ontology and implementing multi-agent technology of collective decision making for supporting digital twins of plants.

Predefined part of digital twin of plant can be combined in CP MAS with accumulating data and machine learning ability for extracting hidden patterns and dependencies for predicting plant reactions to changes in weather conditions or farmers actions, including choice of technology, intensification of fertilizing, etc.

The proposed CP MAS could be used both in daily management of plant growing and also for simulation of perspective scenarios for the new seasons, forecasting yields and risks for business.

Development of an CP MAS for precise farming can help to collect, systemize and formalize, visualize and objectify decisions that are critical for business and make them more efficient, reliable and argumented.

The functionality of designed CP MAS can provide the following benefits for farmers:

1. collect everyday data on plant cultivation and provide recommendations;
2. improving the quality and efficiency of farm management decisions;

3. possibility of more objective, accurate, reliable and reasonable farm business planning and control of execution;

4. continuous extraction and formalization of enterprise knowledge for decision making based on analysis of incoming data and adjusting computational models of plants;

5. reducing dependence on agronomists personalities and negative human factors;

6. providing scalable platform for business developments.

The developed "digital twins" of plants will help to accumulate and formalize new valuable knowledge for agronomists and provide more accurate planning of technological operations in crop cultivation.

In the future, the developed solution could become the core part of an autonomous CP MAS for resource management in precise farming.

\section{Acknowledgements}

The work was supported by the Ministry of Education and Science of the Russian Federation in the framework of contract agreement with Samara State Technical University No. 05.604.21.0224 (lot 2019-05-576-0001, submission number: 2019-05-576-0001-133) - the unique ID number is RFMEFI60419X0224. The project title: "The development of design principles, models, methods and tools to provide functionality of smart cyberphysical system for managing agricultural enterprise of precise farming based on digital twins of plants".

\section{References}

Dutta, S., Lanvin, B., and Wunsch-Vincent, S., editors (2017). The Global Innovation Index 2017: Innovation Feeding the World. Cornell, INSEAD and WIPO, Ithaca, Fontainebleau and Geneva.

Leitao, P., Karnouskos, S., Ribeiro, L., Lee, J., Strasser, T., and W.Colombo, A. (2016). Smart agents in industrial cyber-physical systems. Proc. IEEE, 104(5), pp. 1086-1101.

Qi, Q. and Tao, F. (2018). Digital twin and big data towards smart manufacturing and industry 4.0: 360 degree comparison. IEEE Access, 6, pp. 3585-3593.

Rajkumar, R., Lee, I., Sha, L., and Stankovic, J. (2010). Cyber-physical systems: The next computing revolution. In 47th ACMIEEE Des. Autom. Conf. DAC, pp. 731-736.

Rzevski, G. and Skobelev., P. (2014). Managing Complexity. WIT Press, London-Boston.

Tao, F., Cheng, J., Qi, Q., Zhang, M., Zhang, H., and Sui, F. (2018). Digital twin-driven product design, manufacturing and service with big data. Int. J. Adv. Manuf. Technol., 94 (4), pp. 3563-3576. 